\title{
Magnetic Particle Imaging Using Moving Halbach Cuboid and Frequency Mixing Magnetic Detection
}

Jaechan Jeong

Electronics and Telecommunications Research Institute

Jinsun Kim

Electronics and Telecommunications Research Institute

Beomsu Seo

Electronics and Telecommunications Research Institute

Hans Krause

Forschungszentrum Jülich

Hyobong Hong ( $\sim$ hb8868@etri.re.kr)

Electronics and Telecommunications Research Institute

\section{Research Article}

Keywords: magnetic particle imaging, frequency mixing magnetic detection, superparamagnetic, harmonic frequencies

Posted Date: December 3rd, 2020

DOI: https://doi.org/10.21203/rs.3.rs-113541/v1

License: (c) (i) This work is licensed under a Creative Commons Attribution 4.0 International License.

Read Full License 


\title{
Magnetic Particle Imaging using moving Halbach cuboid and Frequency Mixing Magnetic Detection
}

\author{
Jaechan Jeong ${ }^{\mathrm{a}}$, Jinsun Kimª, Beomsu Seo ${ }^{\mathrm{a}}$, Hans-Joachim Krause ${ }^{\mathrm{b}}$, \\ and Hyobong Hong ${ }^{\mathrm{a}, *}$
}

\footnotetext{
${ }^{a}$ Electronics and Telecommunications Research Institute (ETRI), 218 Gajeong-Ro, Yuseong-Gu, Daejeon 34129, Republic of Korea

${ }^{\mathrm{b}}$ Institute of Biological Information Processing - Bioelectronics (IBI-3), Forschungszentrum Jülich, Jülich 52425, Germany

*Corresponding author: $\underline{\text { hb8868@etri.re.kr }}$
}

\section{[Abstract]}

We present a magnetic particle imaging (MPI) device using a Halbach cuboid magnet and frequency mixing magnetic detection (FMMD) technology. A Field Free Line was formed in the center of a two-piece Halbach cuboid. Then, the cuboid was moved in the sample volume in a T-shaped and circular shape. The sample was exposed to a magnetic excitation field of two different frequencies. Due to the nonlinearity of the superparamagnetic iron oxide nanoparticles (SPIONs), harmonic frequencies and intermodulation products of the excitation frequencies are generated. This characteristic response signal from the particles was acquired by a coil system and demodulated by a FMMD electronics. Images were created by a backprojection method based on Radon and inverse Radon transformation. Using the Halbach cuboid, we were able to generate a stronger magnetic field compared to the previously reported equipment using large permanent magnets.. The results of the experiment showed that the combination of the Halbach cuboid and FMMD can acquire images similar to those of other existing MPI systems, suggesting that it is a method that has advantages in manufacturing and operation of MPI. 


\section{[Introduction]}

Magnetic Particle Imaging (MPI) technology is one of the technologies of medical imaging equipment that has recently received considerable attention ${ }^{1-4}$. Much work has been done since Gleich and Weizenecker published the first paper on MPI in $2005^{5}$. MPI is a method of measuring the spatial distribution of magnetic nanoparticles and a field in which various kinds of clinical applications are being studied due to the advantage of being able to identify the location of various kinds of diseases because antibodies or antigens can be immobilized on the surface of Nano Magnetic Particles (NMP) ${ }^{1,6-8}$. Since MPI is a non-ionizing method, it has the other advantage of higher safety than radiation-based methods such as Positron Emission Tomography. In relation to the instrumentation of MPI, fabrication can be largely divided into two parts. The first concerns how to obtain the signal from the magnetic nanoparticles. The second is to find out where the signal is generated. The signal of MPI developed so far is based on the nonlinear magnetic properties of NMPs. The core material of magnetic particles used in MPI is mostly iron oxide. Magnetite and maghemite are the most commonly used NMPs among iron oxides. Although the basic magnetic properties of these two iron oxides are ferrimagnetic, when the size of the particles is less than $100 \mathrm{~nm}$, they have superparamagnetic properties, which are good properties as signal materials. So, while the core material is iron oxide, NMPs, which have superparamagnetic properties (SPIOs), are mainly used in many applications including MPI ${ }^{6}$. SPIOs reacts sensitively to changes in the external magnetic field due to its very high magnetic susceptibility, but has a characteristic that it does not exhibit magnetic force when the external magnetic field is removed because remnant magnetization is very small. The magnetic properties of SPIOs can be described by the Langevin function.

$$
\begin{aligned}
& M\left(\mu_{0} H\right)=M s \bullet L\left(\frac{m_{0} \mu_{0} H}{k_{b} T}\right) \\
& L(x)=\operatorname{coth}(x)-\frac{1}{x}
\end{aligned}
$$

$m_{0}$ is the magnetic moment [in $\mathrm{Am}^{2}$ ] of a single magnetic particle, $\mu 0=4 \pi \times 10^{-7} \mathrm{Vs} / \mathrm{Am}$ is the vacuum permeability, $\mathrm{k}_{\mathrm{B}}$ is the Boltzmann constant, $\mathrm{T}$ the temperature in Kelvin, $\mathrm{Ms}$ is the saturation magnetization of the particles, and $x=m_{o} \mu_{0} H / k_{b} T$ denotes the dimensionless (scaled) magnetic field ${ }^{9}$. To summarize briefly, it indicates that the B-field change is non- 
linear when applying a linear change of external magnetic force (H-field) to SPIOs. MPI developed so far is to apply sinusoidal magnetic fields to SPIOs in order to obtain such a characteristic nonlinear magnetic response signals. Two methods are mainly used to apply the sinusoidal field. The first is a method of applying a single frequency and recording the higher harmonic responses, and the second is a method of applying two frequencies and recording frequency mixing responses. The single-frequency signal acquisition method has been used by many research groups since the first paper of MPI ${ }^{2,5,10}$. The theoretical background of mixing the frequency has been proposed by Paoli and Svacek ${ }^{6}$. Based on this theory, other researchers have published papers using this principle as a measurement method for biomaterials ${ }^{9,11-13}$. The brief summary is as follows. When two frequencies (a high frequency component $f 1$ and a low frequency component $f 2$ are applied to SPIOs, a time-varying magnetization is created. When this signal is integrally transformed like FFT, uneven harmonics and additionally occur in addition to excitation components $f 1$ and $f 2$ when there is a nonlinear magnetic object in space (ne: even integer). And, the harmonics of even integer multiples and nof 1 (no: odd integer) disappear due to the symmetry of the signal. Instead, when there is no nonlinear magnetic material present, only excitation components $f 1$ and $f 2$ exist.

In all MPIs proposed so far, information on the location and concentration of SPIOs in space is obtained using a Field Free Line (FFL) or a Field Free Point (FFP). FFL or FFP refers to the region or to the point where the field strength becomes zero. In this region, the SPIOs respond to the sinusoidal excitation field. In contrast, in the remaining region where SPIOs are saturated due to the strong magnetic field, they do not respond to the sinusoidal field. It is important to secure a nonlinear signal using a sinusoidal signal in this area, and make it impossible to obtain a signal due to magnetic saturation of SPIOs outside this area. In addition, whether the created FFL or FFP is effectively moved in the sample volume is another important factor. As for the method of generating and moving FFL or FFP, various methods have been proposed and used by different research teams. In a paper published by Gleich and Weizenecker in $2005^{5}$, the entire process from FFL to signal acquisition was based on solenoid coils. Since then, there has been a lot of research related to it, and it is currently being used in products commercialized jointly by Bruker and Philips (https://www.bruker.com/products/preclinicalimaging/mpi-welcome/brukerphilips.html). Another method is to use a combination of solenoid coils and permanent magnets. This method has been proposed by Goodwill et al. ${ }^{2}$. 
The selection field is made by a permanent magnet and the drive field by a solenoid coil (https://www.magneticinsight.com/). Another method is to create an FFL using a permanent magnet and move it mechanically. This method can be divided into a method using a large magnet and a method using a Halbach magnet array according to the method using a permanent magnet. In the case of large magnets, there is an advantage that the amount of calculation required when designing MPI is very small and can be made in an intuitive form. However, in order to increase the size, it is very difficult to obtain a large magnet with a constant magnetic force on the surface, and safety issues arise during manufacture. In a previous study, we presented a study on a device that can image the spatial distribution of SPIOs by permanent magnets and FMMD technology ${ }^{14,15}$. In this research, instead of using large magnets, an MPI system using Halbach cuboid and detection coils based on FMMD technology was fabricated. A Halbach array is a way to control the direction and intensity of magnetic force by spatially arranging several permanent magnets. A Halbach cylinder is a magnet array in a ring shape. The biggest feature of the Halbach cylinder is that the strength of the magnetic field outside the ring is theoretically almost zero, and the strength of the magnetic field inside and the distribution of the magnetic field can be controlled. FFL generation systems using Halbach cylinders are currently being used frequently in MPI research ${ }^{16,17}$. The method of making the Halbach cylinder proposed so far is to harden a small magnet with epoxy resin while adjusting the angle in a circular frame, or to make a frame using nonferrous metal (mainly aluminum) to fix the positions ${ }^{17-20}$. In reality, it is difficult and dangerous to arrange strong magnets at an angle. Instead, we fabricated a Halbach cuboid that can produce an FFL by arranging magnets only vertically or horizontally. In addition, by using a stage that permits translational " $T$ " and "Round" mechanical movements, the equipment could be manufactured without additionally required components such as drive coil, cooling parts and power supply. A detection coil as commonly used in FMMD experiments is being used in the current system; the drive coil and the three-dimensional detection coil (and the signal receive chain included therein) could be omitted. The experimental MPI setup comprises a combination of Halbach cuboid and FMMD, which are relatively easy to manufacture and allow to image the distribution of magnetic particles in vivo and thus localize diseases. 


\section{[Materials and Methods]}

\section{[MPI-system]}

Figure 1 shows the MPI system used in this study, which is divided into four parts. Halbach cuboid, FMMD coil, mechanical stages, and control box. In order to control the system integratedly, the MFC-based software developed by us was used. The S/W is registered with the following number with the Korean Copyright Association (C-2019-027032).

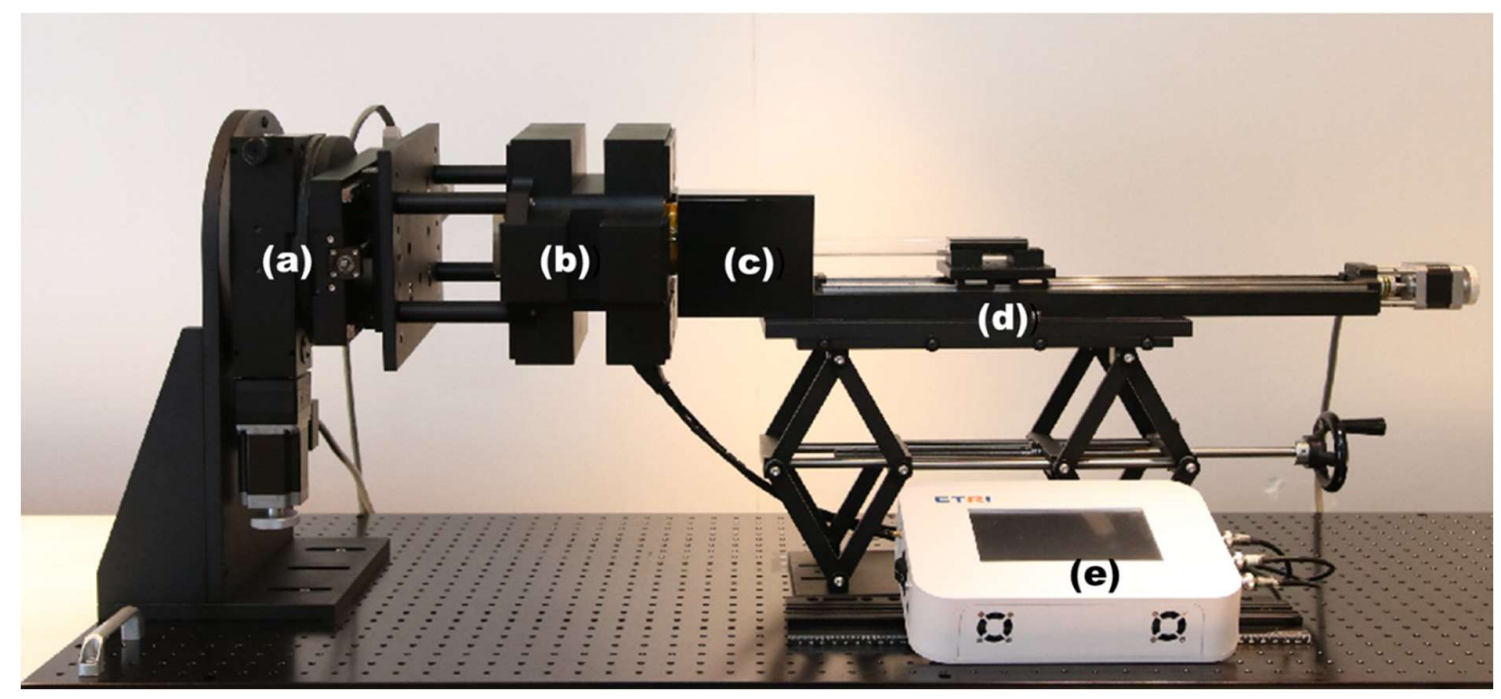

Figure 1. MPI system. (a) stage for T \& Round movement (b) Halbach cuboid (c) FMMD coil (d) linear stage for Z-axis movement (e) control box

Figure 1 (a) and (d) shows the mechanical stages (Namil Optical instruments Co. Incheon, Korea) combining $\mathrm{T}$ and Round stage can perform circular and linear movements simultaneously. The linear stage moves the specimen into the FMMD coil constantly in the Zaxis. More details of the stages are described in a previous publication ${ }^{14}$. The magnet used is $\mathrm{NdFeB}(\mathrm{N} 35$ grade), and the size is $50.0 \times 50.0 \times 25.0 \mathrm{~mm}$. Two magnets stacked together are used as one magnet. The magnetic field gradient strength of the magnet stack is $3.66 \mathrm{~T} / \mathrm{m}$ for $\mathrm{X} \& \mathrm{Z}$ axis. The distribution of magnetic fields in the Halbach cuboid was measured by mounting a Hall sensor (Sensor 2go, Infineon, Munich, Germany) on a 3-axis robot (TT tabletop robot, IAI, Shizuoka, Japan). In order to visually confirm the generation of FFL, it was reconfirmed using a magnetic viewing film (CMS magnetics, Garland, TX, US ). The coil was designed using our simulation software (Coilsimulation.vi). The FMMD measurement heads are composed of three coils each, two excitation coils and one detection coil consisting of two oppositely wound compartments. The excitation coils are wound clockwise (if seen from the 
top) in both measurement heads. They consist of a set of inner and outer coil generating a high and a low frequency magnetic field, respectively. The detection coils are located at the innermost part, and wound clockwise in one half and counter-clockwise in the other half of each measurement head.

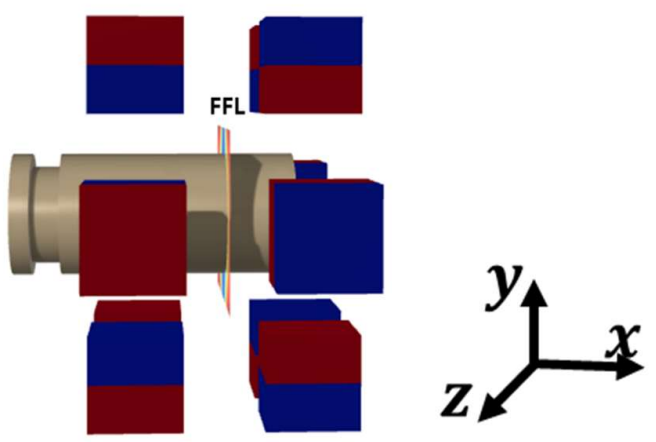

\section{Figure 2. Illustration showing the location of FMMD coil and FFL in the Halbach} cuboid.

Figure 2 shows where the FMMD coil is located in the Halbach cuboid. The $1 / 2$ point of the detection coil divided into two is located in the center of the Halbach cuboid. The FFL is located near the center of one side of the symmetrically wound detection coil. And, as mentioned above, the Halbach cuboid and FMMD coil do not move along the z-axis. Therefore, in order to obtain a three-dimensional image, the specimen is moved to pass the FFL using the Z-axis stage.

Table 1 gives detailed information on this coil system. The bobbin is fabricated from Polyetheretherketone material. The control box (UML-SNAS-100, UMLogics, Daejeon, Korea) used in this study includes a function to apply two frequencies to the FMMD coil and automatically acquire a non-linear signal. In addition, it includes a function to simultaneously acquire location information generated in the used stage. In order to supply sufficient power to the currently used FMMD coil, the low frequency and high frequency channels were connected to an AC amplifier (7224, AE TECHRON, IN, USA) and amplified 20 times. 
Table 1. Parameters of the FMMD coils. DE-detection coil, HF-high frequency coil, LFlow frequency coil.

\begin{tabular}{lccc}
\hline & DE & HF & LF \\
\hline Width & $2 \times 40$ & 101 & 101 \\
Inner radius & 21 & 26.5 & 27.5 \\
Height & 1.75 & 0.81 & 3.81 \\
Wire diameter & 0.3 & 0.4 & 0.4 \\
No. of layers & 6 & 2 & 10 \\
No. of windings & $2 \times 726$ & 464 & 2320 \\
\hline
\end{tabular}

An AC voltage between 120 and $200 \mathrm{~V}$ at a frequency of $75.0 \mathrm{~Hz}$ was applied to the LF coil, and and the same voltage at $7.0 \mathrm{kHz}$ frequency was applied to the HF coil.

\section{[Operation and performance verification for MPI system]}

The SPIO solution was purchased from Micromod (Synomag ${ }^{\circledR}$, Rostock, Germany). The nominal hydrodynamic size of the particle was $50 \mathrm{~nm}$. In this experiment, the pure NMP solution was used as the stock solution and the concentration was $25 \mathrm{mg} / \mathrm{ml}$. The experimental data was obtained by performing a few procedures before acquiring image data. For all experiments, 3 sets of samples were prepared, measured at least 3 times, and the average value and standard deviation of the FMMD signal $f 1+2 f 2$ were recorded. In order to get the negative reference data, the MPI system was operated without a sample, and the signal of $f 1+2 f 2$ was measured. Then, the sample was placed exactly at the center position of the detection coil, which gives the strongest signal. The detection limit for the SPIOs was measured by the following method. Ten $\mu$ l of NMP sample was serially diluted to concentrations of $1 / 2$ to $1 / 64$ of the stock solution. For the spatial resolution experiment, a microcapillary tube (Inner diameter: $1.1 \sim 1.2 \mathrm{~mm}$, wall thickness: $0.2+/-0.02 \mathrm{~mm}$ ) was filled with SPIO stock solution (not diluted), and then samples were arranged at intervals of 10.0 and $5.0 \mathrm{~mm}$.

For the 3D image acquisition experiment, a silicone tube (inner diameter $1.0 \mathrm{~mm}$, outer diameter $2.0 \mathrm{~mm}$ ) filled with SPIOs on an acryl rod at intervals of about $10.0 \mathrm{~mm}$ was prepared.

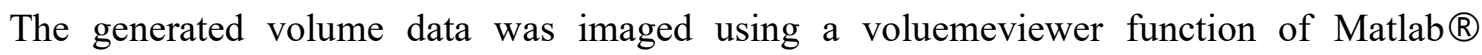
(Mathworks, Natick, MA, USA). In order to prepare Figure 5, the parameters of the rendering editor were set to the maximum intensity projection, the alpha power was set to Mri-Mpi, and 
the color power was set to hot.

\section{[Results and Discussion]}

In this study, we designed and fabricated an MPI system using a Halbach array consisting of relatively small magnets available on the market, replacing the large magnets that were used in our previous work ${ }^{14}$. Since the method used by our research team is to move the Halbach array with the FMMD coil remaining in place, a working distance of about $140.0 \mathrm{~mm}$, which is twice the diameter of the FMMD coil, is required. Therefore, when manufactured in the form of a conventional Halbach cylinder, the total area is 15400.0 square $\mathrm{mm}$. However, since the linear motion itself only needs about $70 \mathrm{~mm}$ in the middle of the rotational motion, there is no need for an area such as this. Therefore, it is optimal to finally go to the cuboid shape by performing simulation on shapes other than the traditional cylinder method. In the case of the cuboid structure, the total area is 9,800 square $\mathrm{mm}$ (smaller than $36 \%$ compared to the cylinder structure). To fabricate an FFL-generating Halbach cuboid, a study on the optimal magnet placement was conducted using electromagnetic field simulation software (Faraday V10.2 Enginia Research Inc, MB, Canada) in advance. Figure 3 and 4 (top) shows that a FFL (Field Free Line) is generated in the center of the structure and the magnetic field gradient within 2.7 $\mathrm{mm}$ from the center of the FFL is $+/-10 \mathrm{mT}$.. 

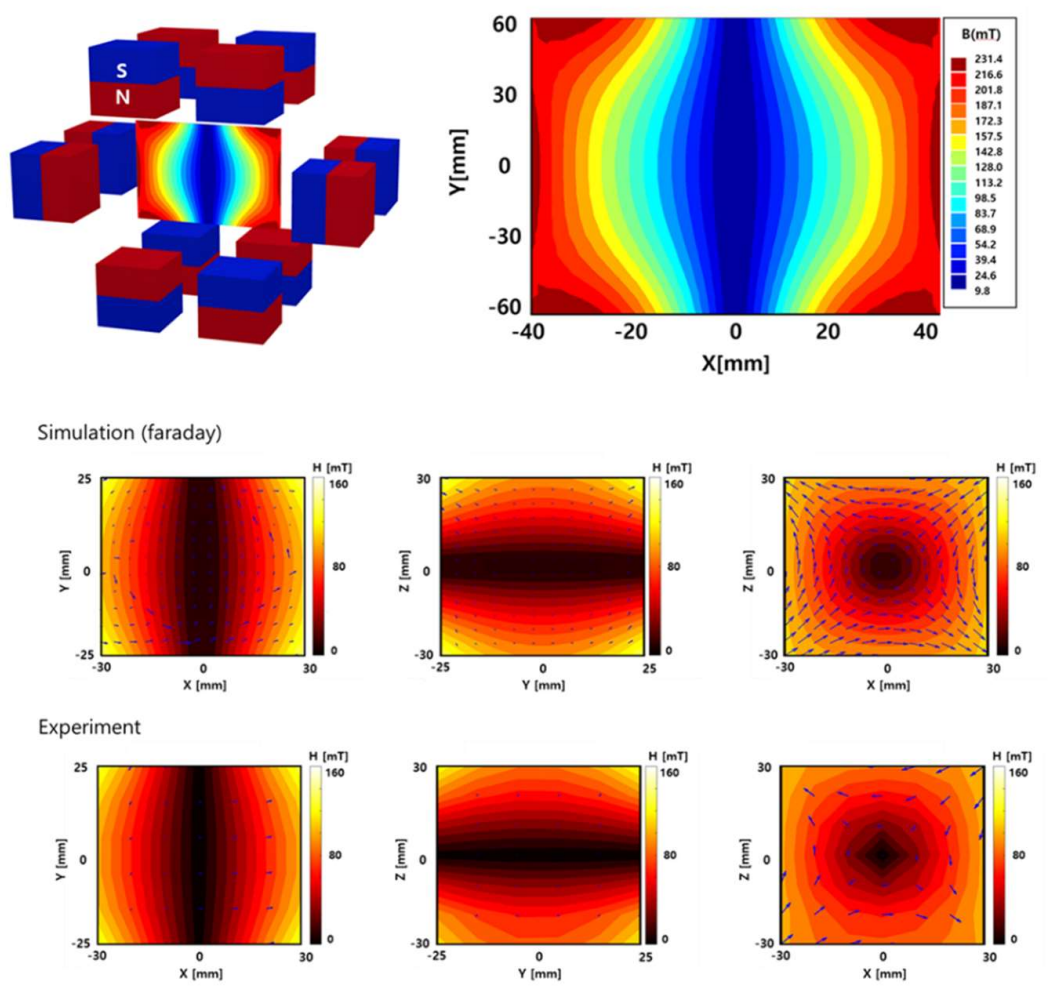

Figure 3. Simulation result of FFL generation according to magnet placement in Halbach cuboid (Top). Distribution of magnetic strength simulated and measured (Bottom)

Based on this simulation, the following structures were fabricated (Figure 3). All of the spherical objects surrounding the magnet were made of aluminum, and then anodizing treatment was performed. In this study, by using a Halbach cuboid instead of the plate-shaped magnet used in our previously published paper ${ }^{14}$, the weight could be reduced to about $1 / 3$ (from $33.0 \mathrm{~kg}$ to $11.0 \mathrm{~kg}$ ), and the strength of the magnetic force was higher than in the MPI using a plate-shaped magnet. It could be increased from $2.9 \mathrm{~T} / \mathrm{m}$ on average to $3.7 \mathrm{~T} / \mathrm{m}$. The magnet shape proposed by other research teams is that many small magnets are solidified with epoxy resin ${ }^{16,17}$, or a Halbach cuboid shape is made by adjusting the angle of a square magnet. This form is typical according to Halbach's theory. However, there are the following problems to be applied in an actual laboratory or commercialization. Even a small magnet has a slight difference in the strength of the magnetic field for each magnet, so even if a theoretically perfect calculation is performed, there are many cases that differ from the calculation in the process of hardening with resin. In reality, it is very difficult to determine the relative and absolute angles of each magnet. However, we were able to minimize various problems required to control the angle and position by placing all magnets vertically or horizontally through simulation. 


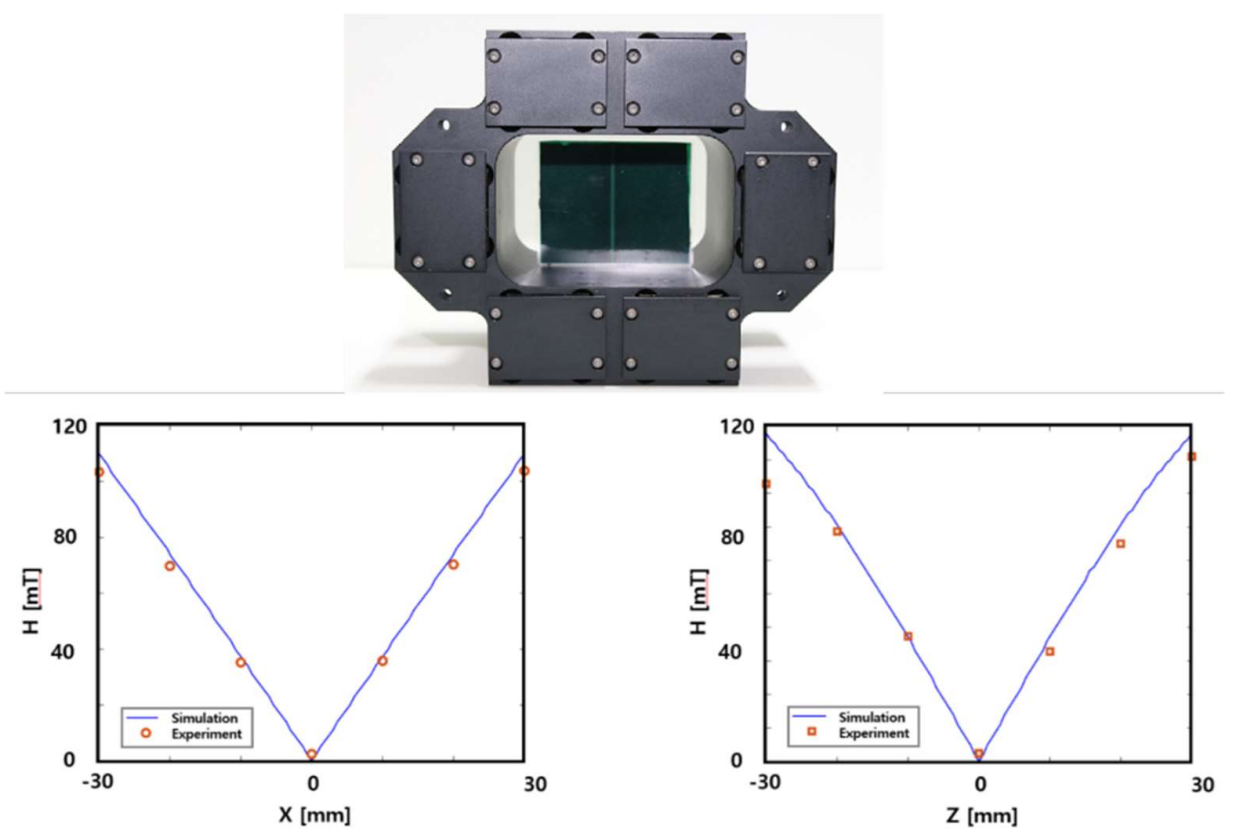

Figure 4. Photograph of Halbach cuboid (Left) Front View, (Middle) Side View and (Left) inside view with the magnets placed.

Figure 5 shows the comparison of the simulated and actual measured values for the magnetic field strength in the manufactured Halbach cuboid. Except for distances of about $30 \mathrm{~mm}$ from the Halbach cuboid surface, the measured values and simulated values are almost identical. Even in the case of an area that is not consistent with the simulation, it does not affect the FFL generation and signal acquisition, and does not affect the results of the experiment. The strength of the magnetic field gradient is $3.7 \mathrm{~T} / \mathrm{m}$ and the aperture size of the Halbach cuboid is $140.0 \times 90.0 \mathrm{~mm}$. Based on this result, the width of the area around the FFL in which the absolute magnetic field is less than $10 \mathrm{mT}$ is $+/-\mathbf{2 . 7} \mathrm{mm}$. The rightmost figure in Figure 4 shows that the actual FFL was created in the center of the Halbach cuboid.. The MPI system using the Halbach cuboid proposed so far uses the method of rotating the cuboid. However, since only rotation can obtain information on angle change, which is one of two sets of information necessary (degree and moving distance), an additional drive coil is installed in most cases ${ }^{17,20}$. In addition, the drive coil installation requires a separate design for the coil and ancillary equipment for power supply and cooling. Therefore, in the method proposed in this study, the function of the drive coil was replaced with a mechanical stage that moves in a "T" shape, as used in our previous setup ${ }^{14}$. The difference from the equipment we presented previously was that a coil was used on one side to provide an aperture for inserting the sample, while the two overlapping Halbach cuboids were used to make even this 
unnecessary. The left figure of Figure 5 shows the imaging results of samples filled with SPIO solution placed side by side with a width of $5.0 \sim 2.0 \mathrm{~mm}$. This result shows that at least $5.0 \mathrm{~mm}$ can be separated and imaged using the currently developed MPI system. The figure on the right shows the test result of whether it is possible to image a sample of a spiral structure, which is a $3 \mathrm{D}$ sample. The results of this experiment show that it is possible to fully image the distribution of three-dimensional samples.

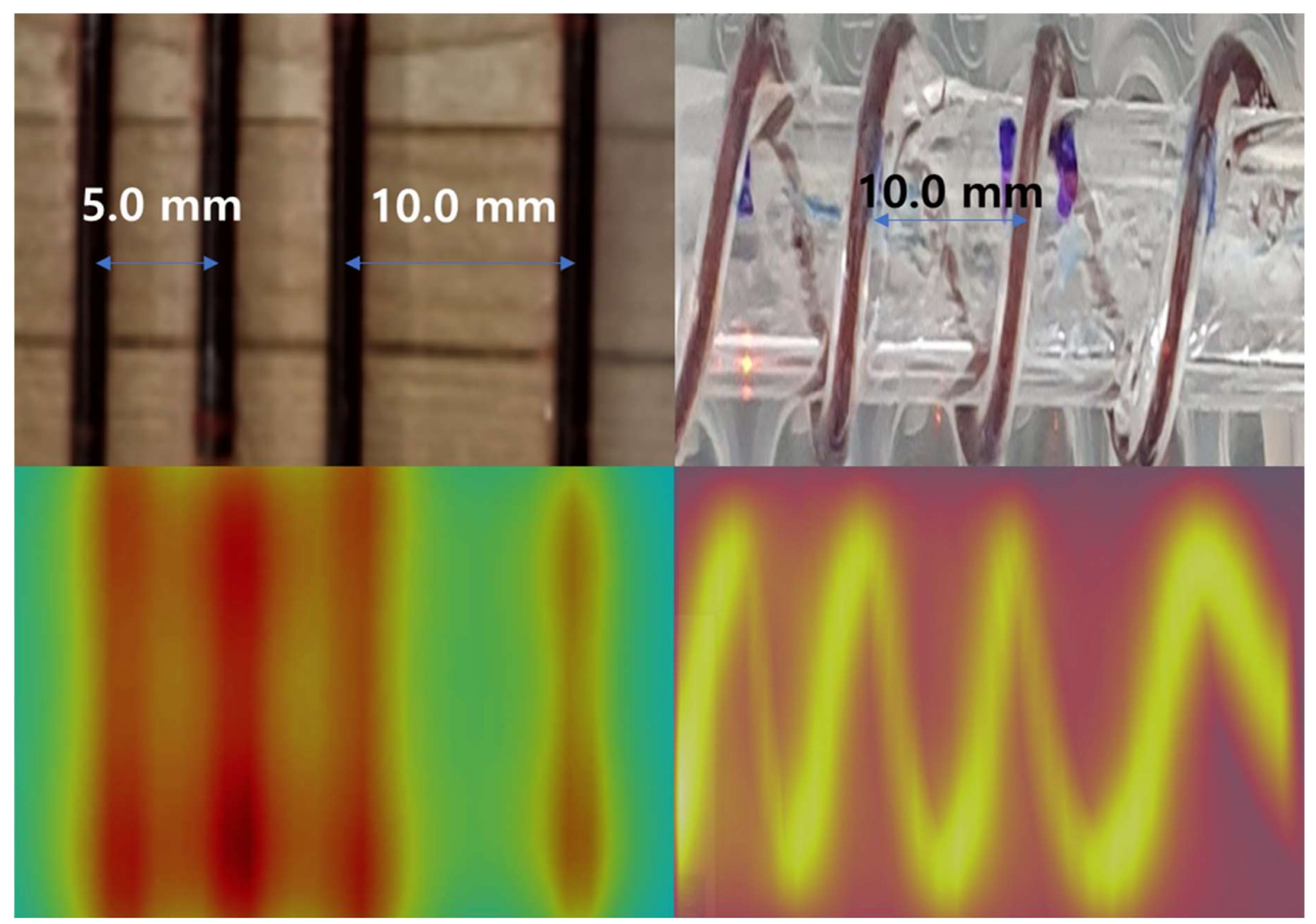

Figure 5. Imaging of capillary tubes placed at intervals of $5.0 \mathrm{~mm}$ and $10.0 \mathrm{~mm}$ (left) and Silicone tube filled with SPIOs wound on an acryl rod. 


\section{[Conclusion]}

In this paper, we introduced a novel approach for manufacturing of MPI equipment, an emerging technology in the medical imaging field, by combining Halbach cuboid, FMMD technology, and stages moving in the "T" and "Round" shapes. When removing the Halbach cuboid, the difficulty in manufacturing was solved by placing the magnet vertically and horizontally, which is not a typical method. Finally, by replacing the production and operation of the drive coil with a stage, it was possible to control the position of the FFL and reduce the need for an additional current supply system. Therefore, this study showed a clear possibility for a new MPI method different from the existing MPI system. 


\section{[Acknowledgement]}

This work was supported by Electronics and Telecommunications Research Institute (ETRI) grant funded by the Korean government [20YS1110]. Our research team is deeply grateful to Dr. Peter Blümler (Institute of Physics, University of Mainz, Germany) for giving much advice on the Halbach magnet.

\section{[Competing Interests]}

The authors declare no competing interests.

\section{[Author Contribution]}

J.J contributed to the production of the equipment and software used in this paper. He also contributed to most of the experiments mentioned in the paper. J.K contributed to the design and manufacture of the Halbach cuboid. In addition, J.K participated in the imaging acquisition experiment and the review of the written paper. H.J.K contributed to the design of the FMMD coil used in this paper and contributed to technical review throughout the paper. B.S assisted the experiment and reviewing manuscript. H.H contributed to the overall concept development and the experiment and wrote the main text.

\section{[References]}

1 Talebloo, N., Gudi, M., Robertson, N. \& Wang, P. Magnetic Particle Imaging: Current Applications in Biomedical Research. Journal of magnetic resonance imaging : JMRI 51, 1659-1668, doi:10.1002/jmri.26875 (2020).

2 Goodwill, P. W. \& Conolly, S. M. The X-Space Formulation of the Magnetic Particle Imaging Process: 1-D Signal, Resolution, Bandwidth, SNR, SAR, and Magnetostimulation. IEEE Transactions on Medical Imaging 29, 1851-1859, doi:10.1109/TMI.2010.2052284 (2010).

3 Knopp, T. et al. Trajectory analysis for magnetic particle imaging. Physics in medicine and biology 54, 385-397, doi:10.1088/0031-9155/54/2/014 (2008).

$4 \quad$ Vogel, P. et al. Magnetic Particle Imaging meets Computed Tomography: first simultaneous imaging. Scientific Reports 9, 12627, doi:10.1038/s41598-019-48960-1 (2019).

5 Gleich, B. \& Weizenecker, J. Tomographic imaging using the nonlinear response of magnetic particles. Nature 435, 1214-1217, doi:10.1038/nature03808 (2005).

6 Chen, Y. T., Kolhatkar, A. G., Zenasni, O., Xu, S. \& Lee, T. R. Biosensing Using Magnetic Particle Detection Techniques. Sensors (Basel) 17, doi:10.3390/s17102300 (2017).

7 Wu, L. C. et al. A Review of Magnetic Particle Imaging and Perspectives on Neuroimaging. 
American Journal of Neuroradiology, doi:10.3174/ajnr.A5896 (2019).

8 Bulte, J. W. M. Superparamagnetic iron oxides as MPI tracers: A primer and review of early applications. Advanced drug delivery reviews 138, 293-301, doi:10.1016/j.addr.2018.12.007 (2019).

9 Krause, H.-J. et al. Magnetic particle detection by frequency mixing for immunoassay applications. Journal of Magnetism and Magnetic Materials 311, 436-444, doi:https://doi.org/10.1016/j.jmmm.2006.10.1164 (2007).

10 Gleich, B., Weizenecker, J. \& Borgert, J. Experimental results on fast 2D-encoded magnetic particle imaging. Physics in medicine and biology 53, N81-N84, doi:10.1088/00319155/53/6/n01 (2008).

11 Meyer, M. H. F. et al. Francisella tularensis detection using magnetic labels and a magnetic biosensor based on frequency mixing. Journal of Magnetism and Magnetic Materials 311, 259-263, doi:https://doi.org/10.1016/j.jmmm.2006.10.1175 (2007).

12 Hong, H., Lim, J., Choi, C.-J., Shin, S. \& Krause, H. Magnetic particle imaging with a planar frequency mixing magnetic detection scanner. The Review of scientific instruments $\mathbf{8 5} \mathbf{1}$, 013705 (2014).

13 Hong, H.-B. et al. Detection of two different influenza A viruses using a nitrocellulose membrane and a magnetic biosensor. Journal of immunological methods 365, 95-100, doi:10.1016/j.jim.2010.12.005 (2011).

14 Choi, S.-M. et al. A novel three-dimensional magnetic particle imaging system based on the frequency mixing for the point-of-care diagnostics. Scientific Reports 10, 11833, doi:10.1038/s41598-020-68864-9 (2020).

15 Kim, C. B., Lim, E. G., Shin, S. W., Krause, H. J. \& Hong, H. Magnetic immunoassay platform based on the planar frequency mixing magnetic technique. Biosensors \& bioelectronics 83, 293-299, doi:10.1016/j.bios.2016.04.076 (2016).

16 Bakenecker, A. C. et al. A concept for a magnetic particle imaging scanner with Halbach arrays. Physics in medicine and biology, doi:10.1088/1361-6560/ab7e 7e (2020).

17 Weber, M. et al. Novel Field Geometry Using Two Halbach Cylinders for FFL-MPI. doi:10.18416/IJMPI.2018.1811004 (2018).

18 Bagheri, H. et al. A mechanically driven magnetic particle imaging scanner. Applied Physics Letters 113, 183703, doi:10.1063/1.5052646 (2018).

19 Halbach, K. Design of permanent multipole magnets with oriented rare earth cobalt material. Nuclear Instruments and Methods 169, 1-10, doi:https://doi.org/10.1016/0029554X(80)90094-4 (1980).

20 Murase, K., Hiratsuka, S., Song, R. \& Takeuchi, Y. Development of a system for magnetic particle imaging using neodymium magnets and gradiometer. Japanese Journal of Applied Physics 53, 067001 (2014). 


\section{Figures}

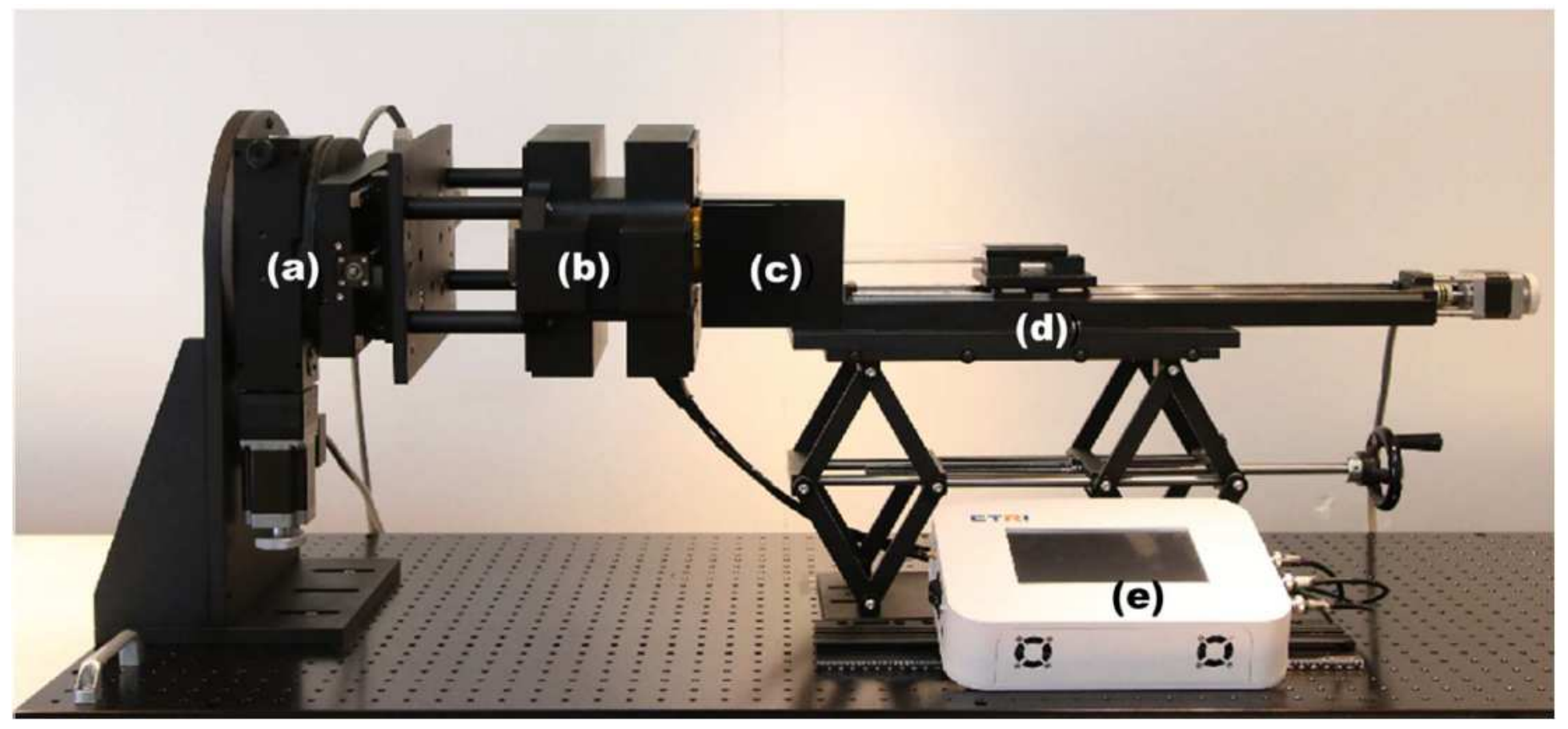

Figure 1

MPI system. (a) stage for T \& Round movement (b) Halbach cuboid (c) FMMD coil (d) linear stage for Zaxis movement (e) control box

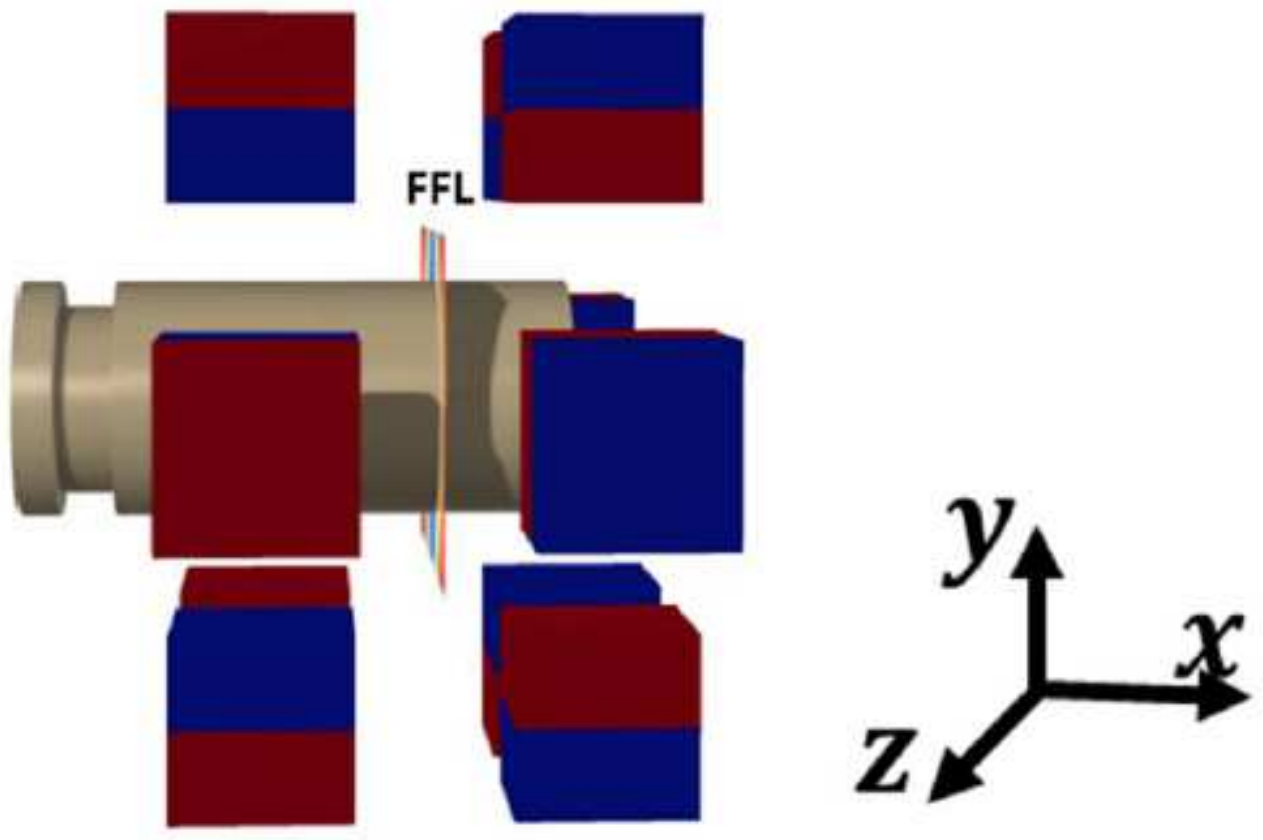

Figure 2

Illustration showing the location of FMMD coil and FFL in the Halbach cuboid. 


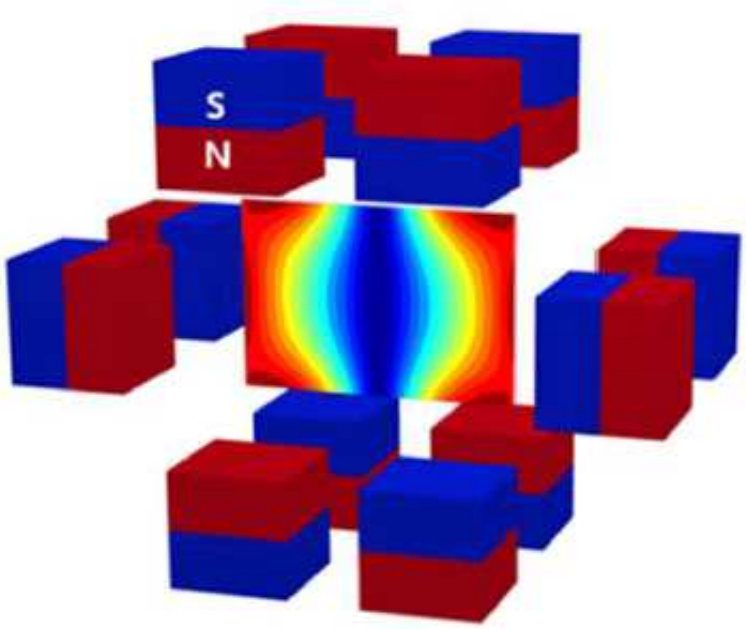

Simulation (faraday)

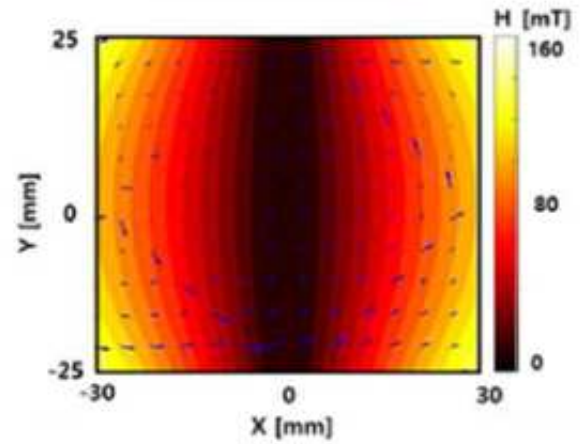

Experiment

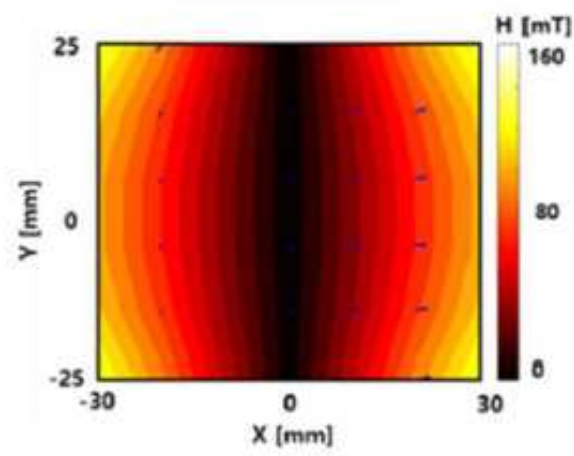

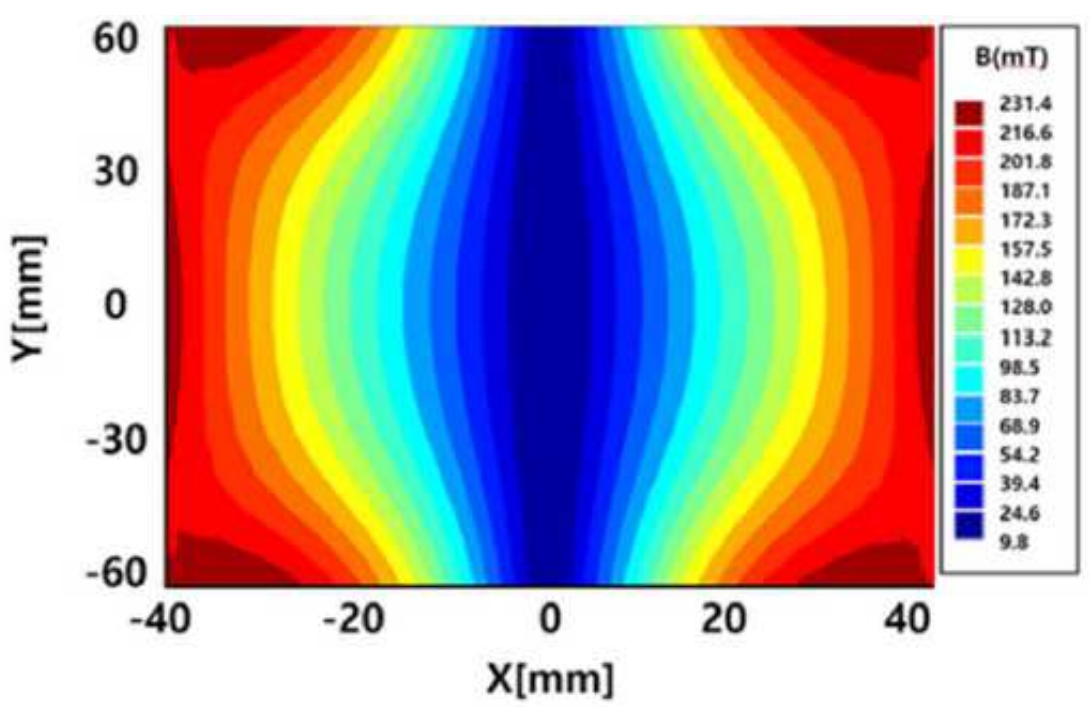
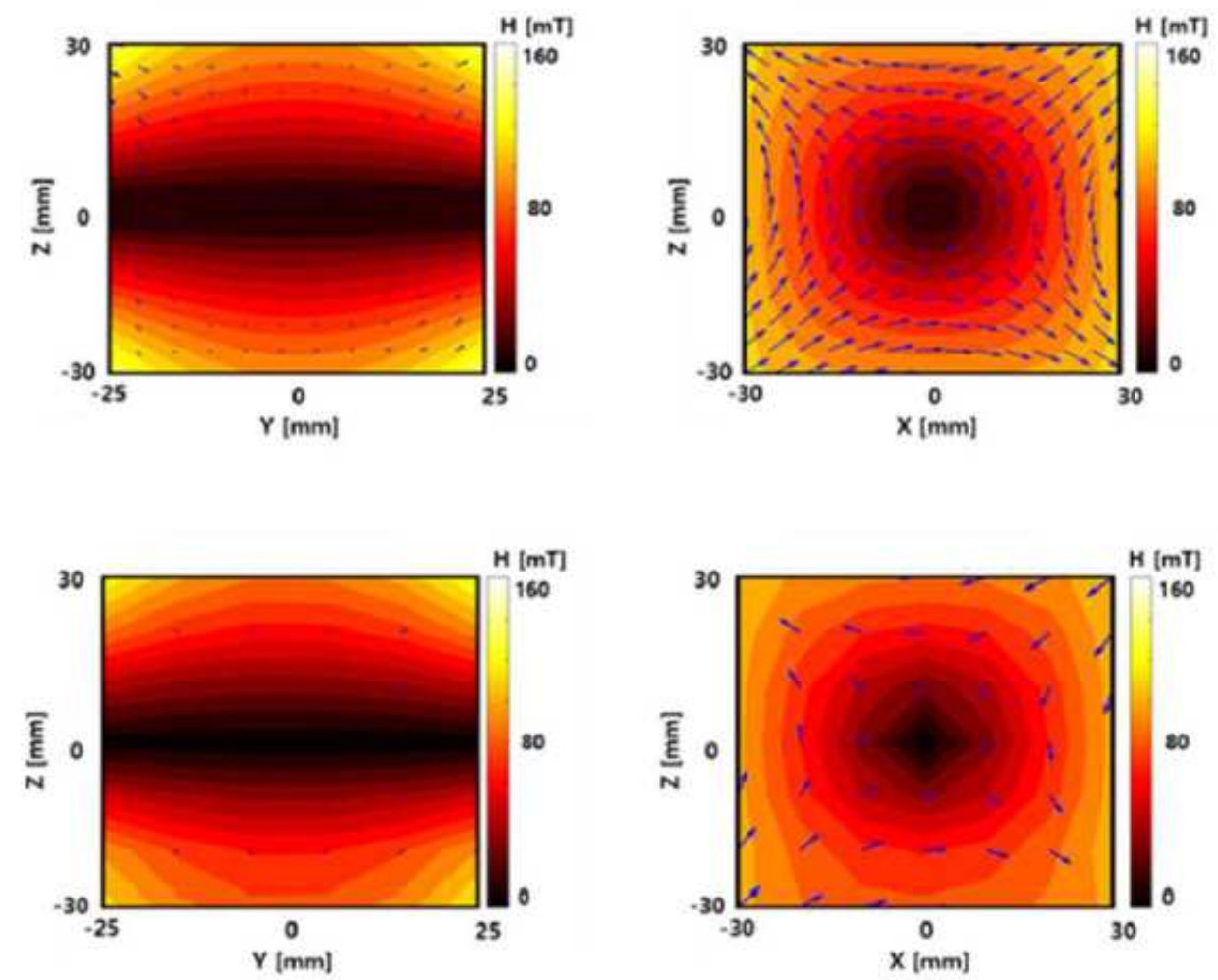

\section{Figure 3}

Simulation result of FFL generation according to magnet placement in Halbach cuboid (Top). Distribution of magnetic strength simulated and measured (Bottom) 


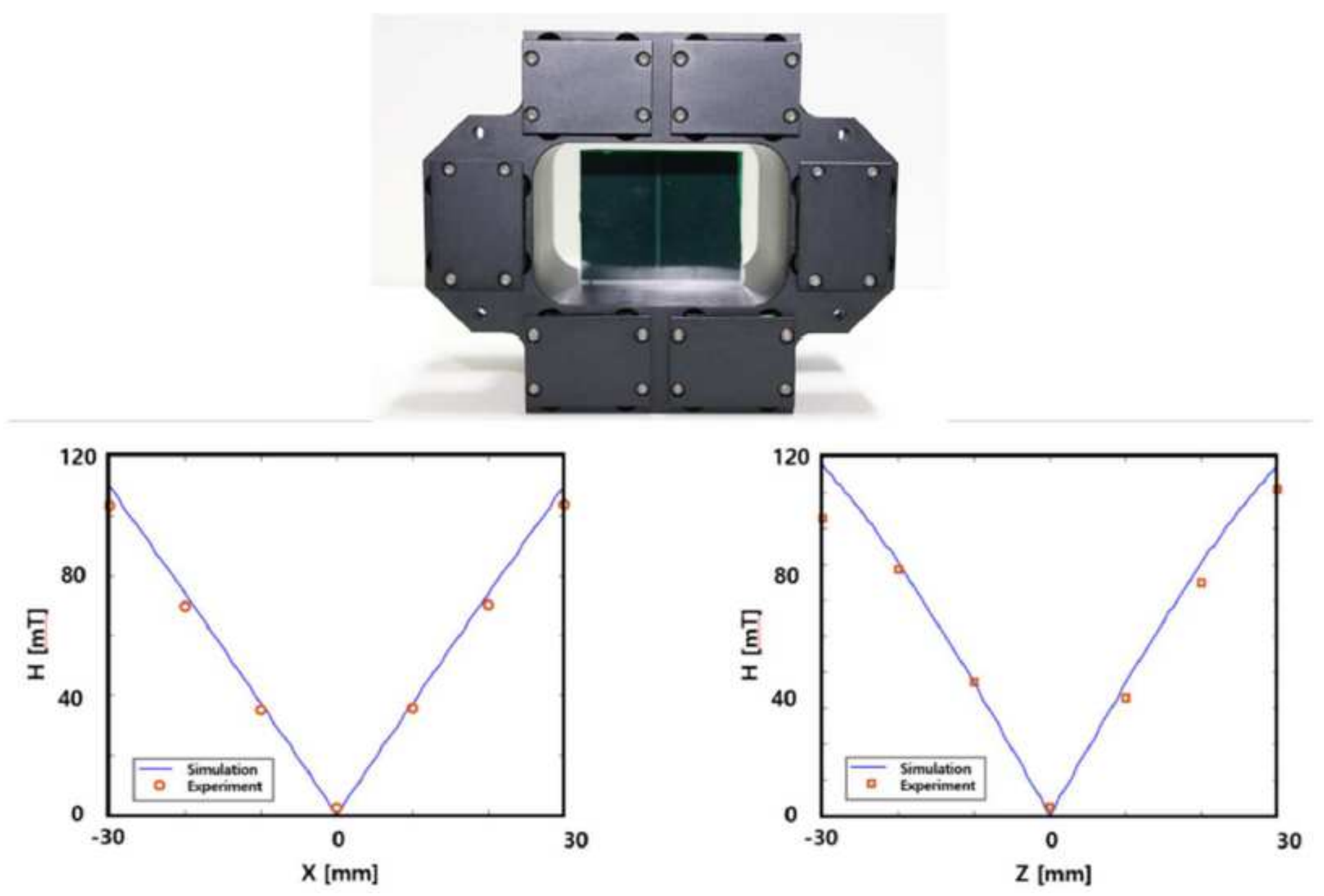

Figure 4

Photograph of Halbach cuboid (Left) Front View, (Middle) Side View and (Left) inside view with the magnets placed. 


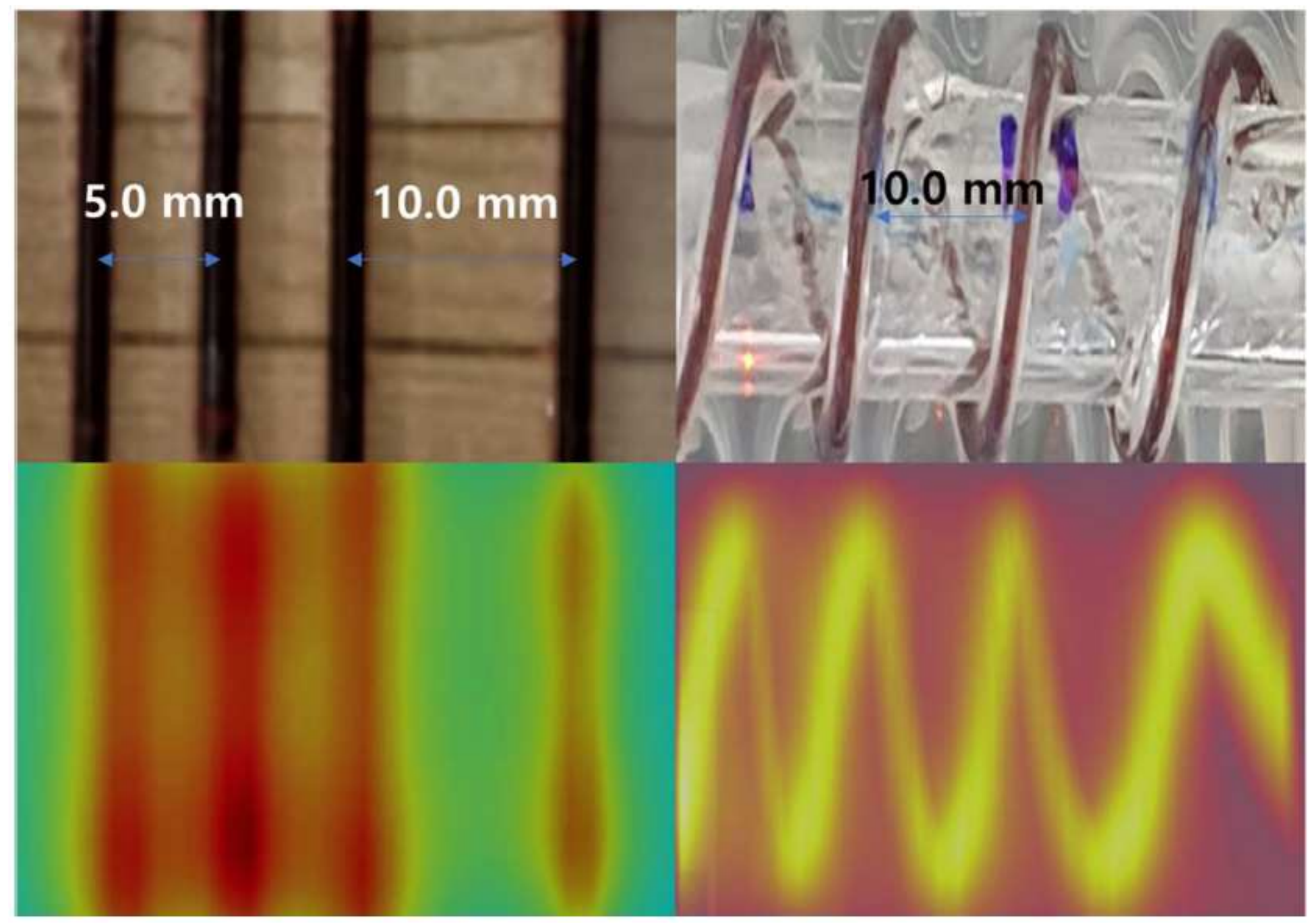

Figure 5

Imaging of capillary tubes placed at intervals of $5.0 \mathrm{~mm}$ and $10.0 \mathrm{~mm}$ (left) and Silicone tube filled with SPIOs wound on an acryl rod. 